\title{
Elastic deformation of the mandibular jaw revisited-a clinical comparison between digital and conventional impressions using a reference
}

\author{
Alexander Schmidt ${ }^{1}$ (D) $\cdot$ Leona Klussmann ${ }^{1} \cdot$ Maximiliane A. Schlenz ${ }^{1} \cdot$ Bernd Wöstmann $^{1}$
}

Received: 31 July 2020 / Accepted: 4 January 2021 / Published online: 13 January 2021

(C) The Author(s) 2021

\begin{abstract}
Objectives Due to the partly strongly differing results in the literature, the aim of the present study was to investigate a possible deformation of the mandible during mouth opening using an intraoral scanner (IOS) and a conventional impression for comparison with a reference aid.

Materials and methods Four steel spheres were reversibly luted in the mandibular $(n=50)$ with a metallic reference aid at maximum mouth opening (MMO). Two digital impressions (Trios3), at MMO and at slightly mouth opening SMO and a conventional impression (Impregum), were taken as the measuring accuracy of the reference structure was already known. Difference between MMO-SMO for digital impressions and deviations between digital and conventional (SMO) were calculated. Furthermore, the angle between the normal vectors of two constructed planes was measured. Statistical analysis was performed with SPSS25.

Results Deviations for linear distances ranged from $-1 \pm 3 \mu \mathrm{m}$ up to $17 \pm 78 \mu \mathrm{m}$ (digital impressions, MMO-SMO), from $19 \pm$ $16 \mu \mathrm{m}$ up to $132 \pm 90 \mu \mathrm{m}$ (digital impressions, SMO), and from $28 \pm 17 \mu \mathrm{m}$ up to $60 \pm 52 \mu \mathrm{m}$ (conventional impressions, SMO). There were no significant differences for digital impressions (MMO-SMO), and there were significant differences between the conventional and digital impressions at SMO.

Conclusions Based on the results of the present study, no mandibular deformation could be detected during mouth opening with regard to the digital impressions. The results were rather within the measuring tolerance of the intraoral scanner.

Clinical relevance Based on the present study, no deformation of the mandibular during mouth opening could be observed at the level previously assumed. Therewith related, dental techniques related to a possible mandibular deformation therefore should be reconsidered.
\end{abstract}

Keywords Clinical study $\cdot$ Digital dentistry $\cdot$ Dental impression technique $\cdot$ Full-arch impression $\cdot$ Dimensional measurement accuracy $\cdot$ Mandibular prosthesis

\section{Introduction}

The elastic deformation of the mandible during mouth opening has been a topic discussed for several decades. Moreover, this discussion continues to influence the education of dental students, and the daily work of dentists in routine dental practice and dental technicians in laboratories, especially when it

Alexander Schmidt

alexander.schmidt@dentist.med.uni-giessen.de

1 Department of Prosthodontics, Dental Clinic, Justus Liebig University, Schlangenzahl 14, 35392 Giessen, Germany comes to wide-span restorations. Therefore, for long-span fixed restorations in the mandible, split bridges are still fabricated to counteract the mandibular deformation during wide mouth opening [1-3].

However, most of the available data rely on studies from the last century [1, 4-11], which is the decisive aspect-show an extremely high variance with respect to the magnitude of the deformation observed. Goodkind and Herinlake found deviations ranging from 32 to $77 \mu \mathrm{m}$ [7], whereas McDowell and Regli detected deviations up to $1400 \mu \mathrm{m}$ [5].

Additionally, in many cases, the data are based on simple measuring devices and techniques. Although the approaches are relatively precise in their nature, they all are confronted with the problem of determining the exact situation in the 
mouth. Thus, most publications rely on the comparison of models made from conventional impressions (CVIs) recorded at different degrees of mouth opening $[5,8,9,11,12]$.

Because these methods are not as precise as the modern digital techniques [6], the available data are contradictory. In addition, during impression taking and model fabrication, there could be possible errors in transfer accuracy already present in the impressions and models. Hence, for a precise evaluation of the transfer accuracy, an intraoral reference is necessary [13-15].

Due to the relatively different results in the literature, the aim of this study was to investigate the possible deformation of the mandible during mouth opening using a reference aid for comparison of between impressions with an intraoral scanner (IOS) and conventional technique.

The null hypothesis was that there is no statistical difference in the accuracy of the mandibular deformation measurement (trueness and precision) with respect to linear distances and possible torsions during mouth opening. A possible influence of female or male participants was investigated as covariate.

\section{Materials and methods}

This clinical study included 50 volunteer participants (aged 18-36 years) with completely dentulous mandibles. Only those individuals with a minimal mouth opening capacity of $37 \mathrm{~mm}$ (incisal edge distance) were included, and the opening capacity ranged from 37 to $64 \mathrm{~mm}$. The investigation was conducted in full accordance with the applicable ethical principles, including that of World Medical Association's Declaration of Helsinki. The present study was approved by the local Ethics Committee of the Justus Liebig University (Giessen, Germany; Ref. no. 163/15). To ensure comparable test results [16], a single operator (L.K.) with experience in digital and conventional impression techniques performed all experiments.

For measuring purposes, four bearing steel spheres (diameter $5000 \pm 5,63 \mu \mathrm{m} ; 1.3505$ 100Cr6 DIN5401 [17], ISO3290-01 [18]; TIS GmbH, Gauting, Germany) were reversibly luted to the mandibular teeth at MMO, using a flowable composite without prior etching (Grandio Flow, Voco, Cuxhaven, Germany, Fig. 1). A metallic reference aid (Bretthauer GmbH, Dillenburg, Germany) [14, 15] was used for exact positioning of the spheres, and a cheek retractor (Optragate, Ivoclar Vivadent, Schaan, Lichtenstein) was used to retract the cheeks and lips. The reference aid used was previously checked for fit in all participants. If the reference aid could not be positioned safely, the participant was excluded from the study.

This method is based on a previously described [14] and investigated technique [15]. In contrast to the previous study
[14], high-precision spheres (roundness $5000 \pm 5.63 \mu \mathrm{m}$ ) were used $[17,18]$. Thus, it was possible to enhance the precision of the placement of the luted spheres from $15 \mu \mathrm{m}$ to less than $10 \mu \mathrm{m}$.

Subsequently, in every volunteer, two digital full-arch impressions were recorded using the Trios 3 Cart wired (software version 19.2.4, normal scan speed mode, manufactured 2016-03, 3Shape, Copenhagen, Denmark) at MMO and SMO. To obtain the best possible scan results under standardized conditions, the IOS systems were calibrated according to the manufacturer's instructions. Scanning was started from the occlusal surfaces of the lower right quadrant to the lower left quadrant, followed by the oral surfaces and then the buccal surfaces [19]. The scan data were directly exported in a standard tessellation language (STL) dataset.

After removing the cheek retractor, a CVI was taken at SMO using a medium-body polyether impression material (Impregum Penta Soft Quick, batch no. 4811262, 3 M, Minneapolis, MN, USA) and a standard metal tray (Ehricke stainless steel, Orbis Dental, Münster, Germany). Before casting with type IV dental stone (Fujirock EP, batch no. 1810031, GC Corporation, Tokyo, Japan), the polyether impressions were stored for at least $2 \mathrm{~h}$ to ensure elastic recovery. The plaster models were stored under laboratory conditions (temperature, $23 \pm 1{ }^{\circ} \mathrm{C}$ and humidity $50 \pm 10 \%$ ) for a minimum of 5 days.

To determine the dimensions of the reference aid, a coordinate measurement machine (CMM) (Thome Präzision $\mathrm{GmbH}$, Messel, Germany) was used with the corresponding software (X4 V10 GA × 64, Metrologic Group, Meylan, France). For the reference dataset, the reference aid with the inserted spheres was measured 10 times, and the mean value for each sphere position was calculated. This digital reference model was stored as a dataset in the IGES format. Thereafter, each plaster model with respective spheres of the CVIs were measured 10 times with CMM, the mean value for each sphere was calculated and saved as digital datasets. The STL datasets of the digital impressions were imported into threedimensional analysis software (GOM Inspect 2019, Gom $\mathrm{GmbH}$, Braunschweig, Germany) for linear measurement between the centers of the spheres. The reference dataset of the reference aid was imported and saved as CAD-data in the GOM software. The imported STL dataset was saved as actual data. As the imported scan dataset only consists of a linked point cloud, four spheres were constructed using fitting elements (Gauß best fit, 3 Sigma) according to the scanned spheres. Subsequently, the deviations between the measured distances of the scan datasets and the reference aid data were calculated. The deviations in the MMO and SMO measurements were subtracted from the reference aid dimensions to obtain the relative deviation during mouth opening.

Furthermore, a possible torsion during mouth opening was measured, and the angle in between the normal vectors of two 
Fig. 1 Fixation of the steel spheres using a metallic reference aid (at maximum mouth opening; left), and luting of steel spheres to the mandible (right)

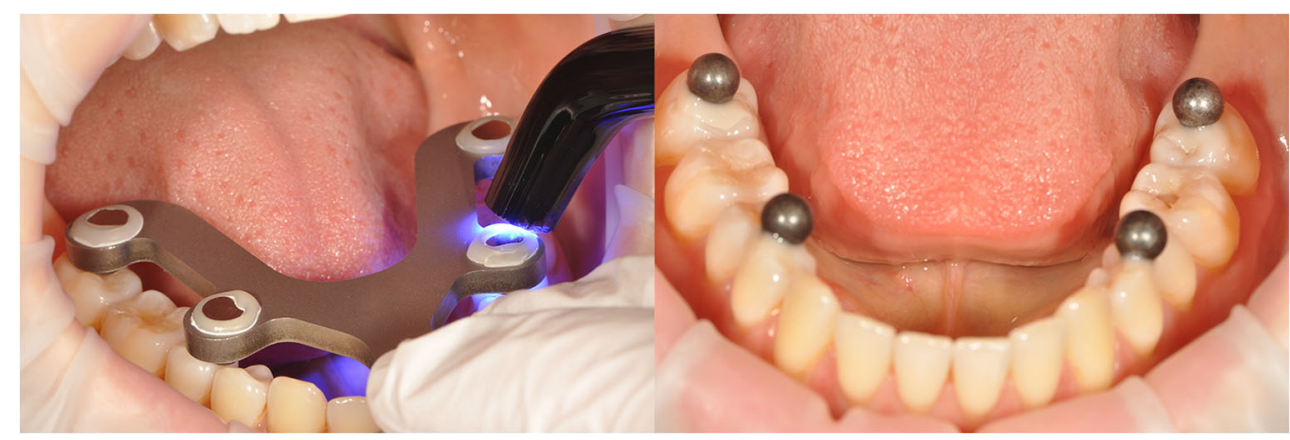

constructed planes was measured (defined by spheres 1,2 , and 4 and 1, 3, and 4; Fig. 2).

Summarized, a reference aid was luted at maximum mouth opening (MMO), and a digital full-arch impression was recorded with an IOS (Trios3) for MMO and slight mouth opening (SMO). The CVI (Impregum Penta) was taken for SMO, and a gypsum model was fabricated (Fujirock). Figure 3 shows an overview over the entire measurement procedure.

Statistical analysis was performed using SPSS version 25 (IBM Corporation, Armonk, NY, USA). The data were tested for normal distribution (Shapiro-Wilk and Kolmogorov-Smirnov Test, Lilliefors-corrected) and variance homogeneity (Levene test). According to ISO 5725-1, the mean values of the deviations between the impression results and the reference aid data described the trueness (mean) and the standard deviation (SD) described the precision [20]. Paired $t$ tests were used to compare the differences in mean values between the different linear distances and angles under identical conditions (MMO, SMO, and CVI), as well as those between different conditions at identical distances and angles for dependent samples. Differences with $p$ value $<0.05$ were considered statistically significant. The Wilcoxon test was used when the test requirements were significantly violated due to outliers. Furthermore, an effect size $r$ was computed for each difference. The interpretation was based on the suggestions of Kühnel and Krebs $(r<0.2$, weak correlation; $0.2<r>0.5$, medium correlation; and $r>0.5$, strong correlation) [21]. To investigate a statistically significant difference between the genders (female, male) of the subjects, the mean equality of the differences between the two groups was tested using a $T$ test on independent samples and the variance equality was investigated using the Levene test. For a better overview, the results are presented as box plot diagrams.

\section{Results}

The digital impressions for MMO and SMO, and the CVIs for SMO resulted in 150 impressions from 50 volunteers. The calculated relative differences in the linear distances between the digital impressions at MMO and SMO are presented in Fig. 4 and Table 1. The results for the digital and conventional impressions at SMO are presented in Fig. 5 and Table 1.

For a better overview, deviations (mean $\pm \mathrm{SD}[\mu \mathrm{m}]$ ) of the linear distances (D1_2, D1_3, D1_4, D2_3, D2_4, and D3_4) and statistical analysis (p values and effect size $r$ ) are presented in Table 1.

Except for D1_2 and D3_4 measurements, the CVI demonstrated the lowest deviation for all measurements.

For both MMO and SMO, the largest deviations were observed for distance D1_4 (intermolar distance).

The lowest deviations in the digital impressions for MMO and SMO were detected for both D1_2 and D3_4 (premolarmolar distances).

There were no statistically significant differences between the deviations at $\mathrm{MMO}$ and $\mathrm{SMO}$ in the digital impressions. However, partially significant differences were observed between the digital and conventional impressions at SMO.

Furthermore, a possible torsion during mouth opening was measured. The angle measured between the normal vectors of the two constructed planes (defined by spheres 1,2, and 4 and
Fig. 2 Representation of the measurement of linear distances (D1_2, D1_3, D1_4, D2_3, D2 4, and D3_4) between the centers of the four spheres (left) and measurement of the angle between the normal vectors (right)
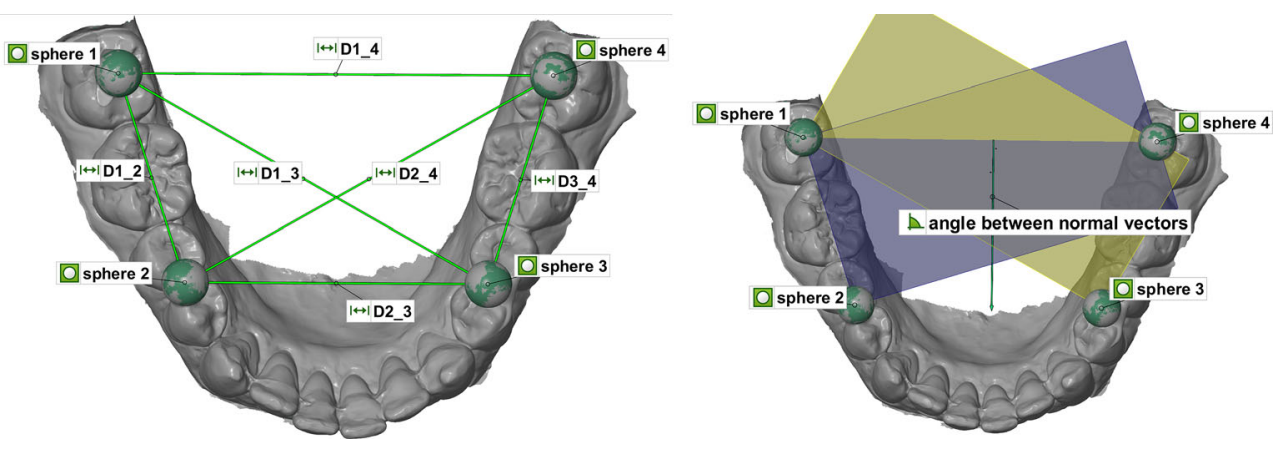
Fig. 3 Overview of the entire measurement procedure

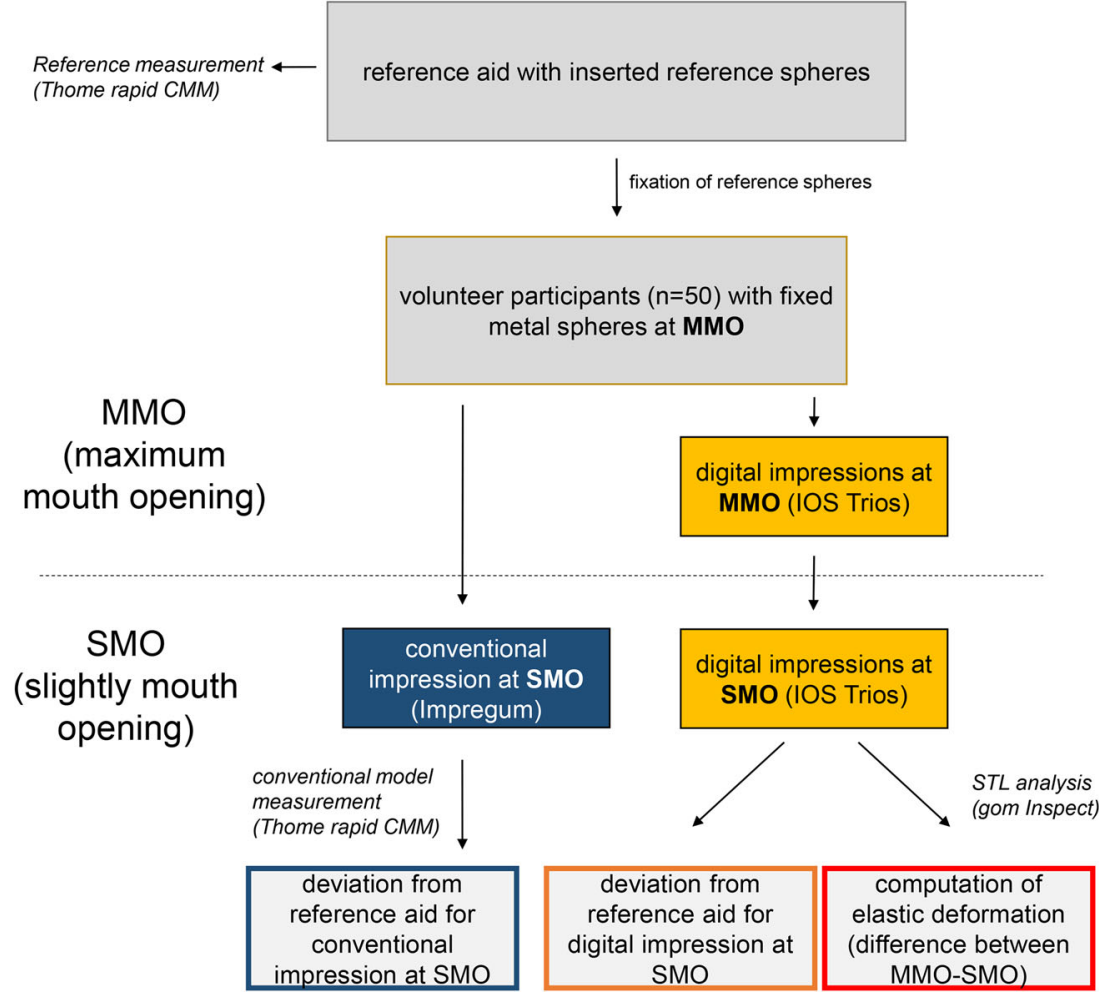

1, 3, and 4) are presented in Fig. 6. For a better overview, deviations in the angle (mean $\pm \mathrm{SD}\left[{ }^{\circ}\right]$ ) and the statistical analysis ( $p$ values and effect size $r$ ) are presented in Table 2.
The largest deviations in the angle at SMO were observed in the digital impressions. Moreover, significant differences were observed between the digital and conventional impressions at SMO.
Table 1 Deviations (mean \pm standard deviation $[\mu \mathrm{m}])$ in the linear distances (D1_2, D1_3, D1_4, D2_3, D2_4, and D3_4) and statistical analysis (significant differences $p<0.05$ and strong correlation standard effect sizes $r$ $>0.05)$ (presented in italics)

\begin{tabular}{|c|c|c|c|c|}
\hline \multirow{2}{*}{$\begin{array}{l}\text { Linear } \\
\text { distances }\end{array}$} & \multirow{2}{*}{$\begin{array}{l}\text { Method/impression } \\
\text { technique }\end{array}$} & \multirow[b]{2}{*}{$\begin{array}{l}\text { Mean (trueness) } \pm \mathrm{SD} \\
\text { (precision) }[\mathrm{mm}]\end{array}$} & \multicolumn{2}{|c|}{$p$ value $/ r$ (effect size) } \\
\hline & & & MMO-SMO & SMO-CVI \\
\hline \multirow[t]{3}{*}{ D1_2 } & MMO-SMO & $0.005 \pm 0.017$ & \multirow[t]{3}{*}{$0.913 / 0.016$} & \multirow{3}{*}{$0.3-$} \\
\hline & SMO & $0.019 \pm 0.016$ & & \\
\hline & CVI & $0.032 \pm 0.023$ & & \\
\hline \multirow[t]{3}{*}{ D1_3 } & MMO-SMO & $0.017 \pm 0.078$ & \multirow[t]{3}{*}{$0.513 / 0.094-$} & \multirow{3}{*}{$\begin{array}{c}<0.001>> \\
0.05\end{array}$} \\
\hline & SMO & $0.076 \pm 0.052$ & & \\
\hline & CVI & $0.038 \pm 0.032$ & & \\
\hline \multirow[t]{3}{*}{ D1_4 } & MMO-SMO & $-0.008 \pm 0.142$ & \multirow[t]{3}{*}{$0.376 / 0.127$} & \multirow{3}{*}{$\begin{aligned}< & 0.001> \\
& 0.05\end{aligned}$} \\
\hline & SMO & $0.132 \pm 0.090$ & & \\
\hline & CVI & $0.060 \pm 0.052$ & & \\
\hline \multirow[t]{3}{*}{ D2_3 } & MMO-SMO & $-0.001 \pm 0.003$ & \multirow[t]{3}{*}{$0.760 / 0.044$} & \multirow{3}{*}{$\begin{aligned}< & 0.001> \\
& 0.05\end{aligned}$} \\
\hline & SMO & $0.065 \pm 0.049$ & & \\
\hline & CVI & $0.034 \pm 0.030$ & & \\
\hline \multirow[t]{3}{*}{ D2_4 } & MMO-SMO & $-0.020 \pm 0.094$ & \multirow[t]{3}{*}{$0.170 / 0.195$} & \multirow{3}{*}{$\begin{aligned}< & 0.001> \\
& 0.05\end{aligned}$} \\
\hline & SMO & $0.095 \pm 0.067$ & & \\
\hline & $\mathrm{CVI}$ & $0.039 \pm 0.029$ & & \\
\hline \multirow[t]{3}{*}{ D3_4 } & MMO-SMO & $0.002 \pm 0.013$ & \multirow[t]{3}{*}{$0.091 / 0.239$} & \multirow[t]{3}{*}{$0.426 / 0.114$} \\
\hline & SMO & $0.025 \pm 0.017$ & & \\
\hline & CVI & $0.028 \pm 0.017$ & & \\
\hline
\end{tabular}


Fig. 4 Box plot diagram of the deviations in the linear distances measured between the centers of the four spheres at maximal mouth opening (MMO) and slight mouth opening (SMO)

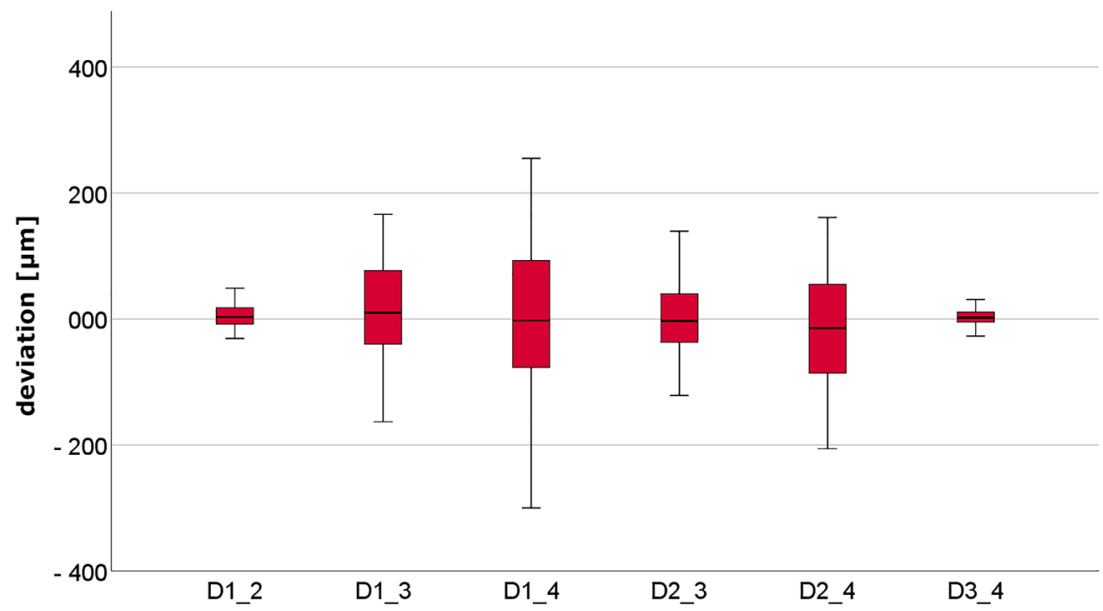

There were no statistically significant differences between $\mathrm{MMO}$ and SMO for the digital impressions.

Statistical analysis of the covariate (female, male subjects) showed no significant differences between the groups.

The null hypothesis that there is no statistical difference in the mandibular deformation accuracy (trueness and precision, ISO 5725 [19]) in linear distances and possible torsions during mouth opening could not be rejected.

\section{Discussion}

For this study, we optimized the method described by Kuhr et al. [14]. Besides the change in the measuring spheres (the previous study [14] used standard spheres as used in implantology) to high-precision spheres with extremely high roundness $[17,18]$, the temperature increase that occurs when changing from room to the oral situation was included in the creation of the reference data set. As the positioning plate is made of stainless steel, the material expands with an increase in temperature. The materialspecific coefficient of thermal expansion was calculated, and the data obtained from the reference measurement with the CMM was corrected by the calculated expansion. In an in vivo study by Kameyama et al. [22], the oral temperature was measured in various participants during an investigation of several intraoral drainage methods. The highest measured temperature was $34^{\circ} \mathrm{C}$, which was confirmed in our preliminary tests. Hence, the oral temperature was fixed at this value and included in the calculation. The greatest thermal expansion for the longest distance (D1_4) was $8 \mu \mathrm{m}$, and this was within the measuring tolerance. Thus, we enhanced the accuracy with which the spheres were luted in place. This is reflected in the results of D1_2 and D3_4 (premolar to molar distance in the left and right quadrants, respectively).

The investigated IOS hardware and software components used in this clinical study are currently available in the market. Before application, the IOS system was calibrated according to the manufacturer's specifications. For better comparison of the results with the current literature, an established methodology was used $[14,15]$, and the results were reported for trueness and precision in accordance with ISO 5725 as described previously in other studies $[13,14,20]$.
Fig. 5 Box plot diagram of the deviations in the linear distances measured between the centers of the four spheres for conventional and digital impressions at slight mouth opening (SMO)

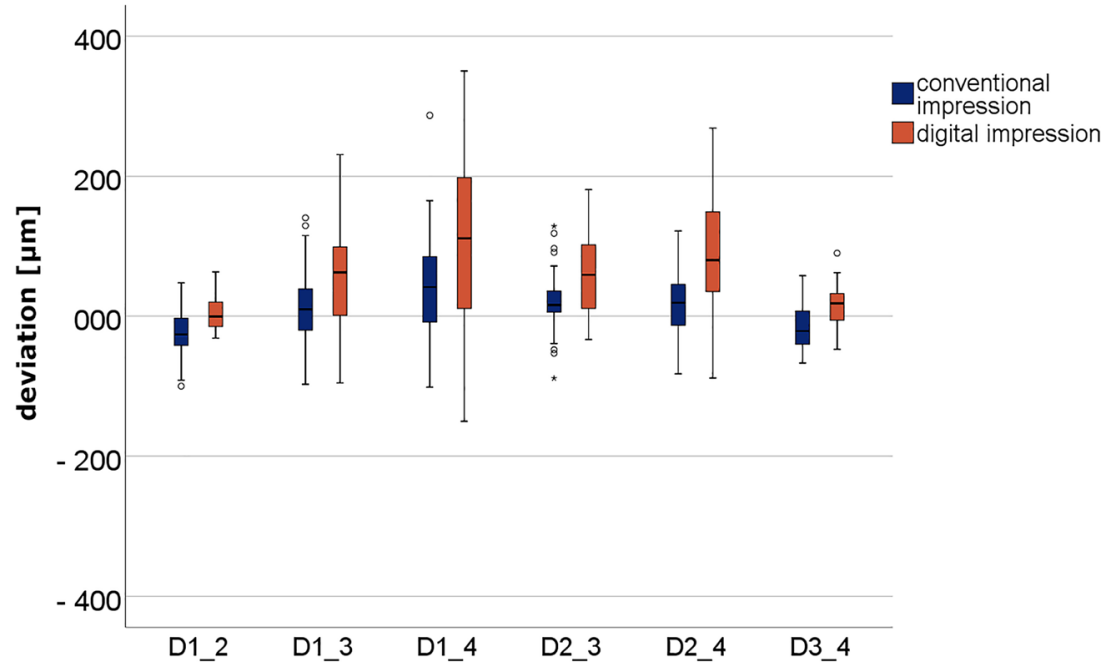


Table 2 Deviations (mean \pm standard deviation $\left[{ }^{\circ}\right]$ ) in the angle between the normal vectors (defined by spheres 1,3 , and 4 and 1,2 , and 4 ) and the statistical analysis (significant differences $p<0.05$ and strong correlation standard effect sizes $r>0.05$ ) (presented in italics)

\begin{tabular}{|c|c|c|c|c|}
\hline \multirow[t]{2}{*}{ Angle between spheres } & \multirow[t]{2}{*}{ Impression technique } & \multirow[b]{2}{*}{ Mean $\pm \mathrm{SD}\left[{ }^{\circ}\right]$} & \multicolumn{2}{|c|}{$p$ value $/ r$ (effect size) } \\
\hline & & & MMO-SMO & SMO-CVI \\
\hline \multirow[t]{3}{*}{$1,3,4$ and $1,2,4$} & MMO-SMO & $-0.046 \pm 0.164$ & \multirow[t]{3}{*}{$0.057 / 0.268$} & \multirow[t]{3}{*}{$<0.001>0.05$} \\
\hline & SMO & $0.25 \pm 0.21$ & & \\
\hline & CVI & $0.11 \pm 0.19$ & & \\
\hline
\end{tabular}

Previous studies described the influence of the scan path on the accuracy of full-arch scans [19, 23, 24]. Therefore, a predetermined scanning protocol was used as recommended by Müller et al., who investigated different scan paths with the IOS Trios3 Pod [19].

With regard to the analysis of accuracy, trueness and precision was assessed according to ISO 5725-1 [20]. Although the method for describing trueness is generally agreed on, different approaches for the assessment of precision have been reported $[25,26]$. We decided to use the ISO approach as a standardized method, which we consider helpful for a later comparison of our results with studies to come [13].

Comparing the results of the present study is difficult because, to our knowledge, no other study has investigated the mandibular deformation using an IOS. Moreover, only three studies have investigated full-arch impressions using a reference aid in patients [13-15]. Recent studies investigating mandibular deformation showed different results. With regard to CVIs, some investigations, such as those by Goodkind and Herinlake [7], showed results similar to that of the present study. They measured an anterior deviation of $32 \mu \mathrm{m}$ and a posterior deviation of $77 \mu \mathrm{m}$. Fischman [27] also presented deviations of $71 \pm 43 \mu \mathrm{m}$, comparable to our study. However, the majority of the studies showed a significantly higher deviation as compared to the results of this study. McDowell and Regli [5] observed MMO deviations of up to $1400 \mu \mathrm{m}$, while
De Marco and Paine [28] observed deviations up to $1500 \mu \mathrm{m}$. Fischman [29] showed an average deviation of $860 \pm 140 \mu \mathrm{m}$ and Prasad et al. [30] from $390 \mu \mathrm{m}$ up to $1120 \mu \mathrm{m}$. Shinkai et al. [12] performed impressions at three different mouth openings and could measure deviations of up to $360 \mu \mathrm{m}$. Nevertheless, these deviations were smaller in a follow-up study [31]. Deviations in the range of 140 up to $300 \mu \mathrm{m}$ could also be shown in investigations of Custodio et al. [32] and Wolf et al. [33]. However, recent investigations were performed with fewer participants, and the measuring methods seemed less precise as compared to those used in the present study. The measuring problem can also be seen on the results of different studies on implants. Hobkirk and Schwab [34], Horiuchi et al. [1], Richter [10], Abdel-Latif et al. [35], and Al-Sukhun and Kelleway [36] fixed the measuring devices in the molar or premolar region on implants. Therefore, smaller deviations could be identified. However, in the case of a possible deformation, exactly the opposite were to be expected on implants, as the deformation that occurred can no longer be compensated by natural teeth.

In contrast, Horiuchi et al. [1] described deviations of two implants from each other at MMO, ranging between 7.8 and $24.6 \mu \mathrm{m}$. Moreover, the linear differences shown in the study by Abdel-Latif et al. [35] ranged between 1.4 and $41.3 \mu \mathrm{m}$, which was reflected in the results of Al-Sukhun and Kelleway [36] who identified deviations of $14.4-58.4 \mu \mathrm{m}$. According to
Fig. 6 Box plot diagram of the deviations in the angle between the normal vectors of the two constructed planes (defined by spheres 1,2 , and 4 and 1,3 , and 4 ) showing difference between digital impressions at maximal mouth opening (MMO) and slight mouth opening (SMO), and between conventional and digital impressions at slight mouth opening

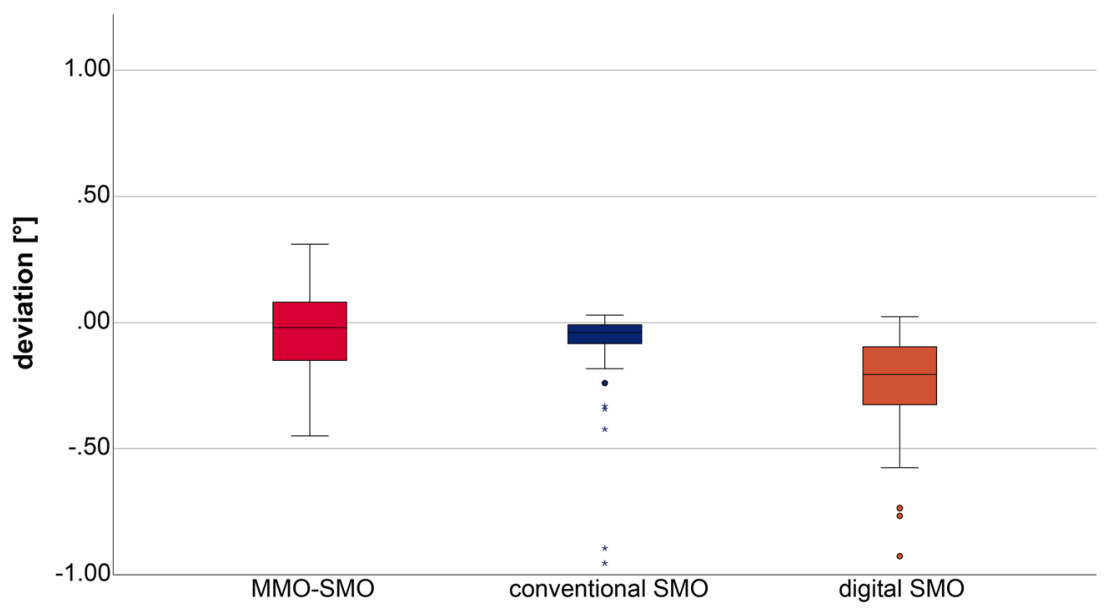


these authors, the deviations could be attributed to a deformation of the mandible during MMO. Because the deviations are in the range of the natural periodontium's own mobility, it is difficult to draw a comparison with the results of the present study.

In this study, no significant difference was detected between the deviations in the female and male participants, and this corroborates with the findings of Chen et al. [37] and Wolf et al. [33].

The deviations measured in the digital impressions for minimum mouth opening were not significantly different from those at MMO. This is clearly elicited in the differences between the results of MMO and SMO (Fig. 4). Here, it is obvious that the median values are almost all on the zero line, and thus no difference between MMO and SMO in terms of mandibular deformation is detectable. Although there is a high dispersion, this could be due to possible stitching or matching errors with increasing scan path length. This is particularly evident in the fact that the short distances of D1_2 and D3_4 hardly showed any deviations. These findings can be compared with the results of a previous study [15]. The longer distances, and also those that completely cross the quadrant (especially D1_4) showed the greatest scatter. These results are comparable with previous investigations [13-15]. In summary, the results of the digital impressions demonstrate that the IOS shows less deviation at short distances up to one quadrant compared to conventional impressions. For fullarch impressions, whether an intraoral scanner can be used for full-arch impressions depends on the definition or indication.

This is confirmed by the deviations in the CVIs. Furthermore, the scattering could be due to plaster expansion and polyether shrinkage, since scattering also occurred in the shorter distances (D1_2 and D3_4). The results were contrasting with the digital impressions of SMO. This confirms the results of a previous study [15] for short distances. Moreover, the spread can be attributed to difference among the patients, as shown by Hobkirk and Schwab [34], Richter [10], and Wolf et al. [33], who reported similar observations. It is noticeable that older investigations where the possible mandibular deformation was measured on models could show very high deviations. This can also be attributed to unavoidable deviations in the fabrication of impressions and plaster casts during extraoral measurements (e.g., plaster expansion and impression material shrinkage) or during intraoral measurements with older measuring instruments $[4,5,28]$.

Thus, the present study does not confirm the previous results of mandibular deformation during mouth opening with deviations up to $1500 \mu \mathrm{m}$. In fact, the results were within the measuring tolerance of the digital and conventional impression methods currently used. Furthermore, it can be said from clinical experience that if deviations of the magnitude described in the literature were to occur, fixed or removable complete prosthetic restorations in the entire mandible would not be possible. As a limitation of the study, possible deformations of the mandible during mouth opening, but not during forward movements of the mandible, were examined.

\section{Conclusions}

In summary, the significant change in the width of the mandible during $\mathrm{MMO}$, which has been partially described in the literature, could not be confirmed in this clinical study. The possible deformations occurring in the mandible were within the possible measuring tolerance of the currently used digital and conventional impression methods.

Acknowledgments We are grateful for the support provided by our biostatistician, Dr. Johannes Herrmann, in performing the statistical analysis in this study.

Funding The work was supported by the Department of Prosthodontics, Justus Liebig University in Giessen, Germany.

\section{Compliance with ethical standards}

Conflict of interest The authors declare that they have no conflict of interest.

Ethical approval All procedures performed in this study involving human participants were in accordance with the ethical standards of the institutional ethic committee and with the 1964 Helsinki declaration and its later amendments or comparable ethical standards.

Informed consent For this type of study, formal consent is not required.

Open Access This article is licensed under a Creative Commons Attribution 4.0 International License, which permits use, sharing, adaptation, distribution and reproduction in any medium or format, as long as you give appropriate credit to the original author(s) and the source, provide a link to the Creative Commons licence, and indicate if changes were made. The images or other third party material in this article are included in the article's Creative Commons licence, unless indicated otherwise in a credit line to the material. If material is not included in the article's Creative Commons licence and your intended use is not permitted by statutory regulation or exceeds the permitted use, you will need to obtain permission directly from the copyright holder. To view a copy of this licence, visit http://creativecommons.org/licenses/by/4.0/.

\section{References}

1. Horiuchi M, Ichikawa T, Noda M, Matsumoto N (1997) Use of interimplant displacement to measure mandibular distortion during jaw movements in humans. Arch Oral Biol 42:185-188. https://doi. org/10.1016/s0003-9969(96)00101-x

2. Korioth TW, Hannam AG (1994) Deformation of the human mandible during simulated tooth clenching. J Dent Res 73:56-66. https://doi.org/10.1177/00220345940730010801 
3. Sivaraman K, Chopra A, Venkatesh SB (2016) Clinical importance of median mandibular flexure in oral rehabilitation: a review. J Oral Rehabil 43:215-225. https://doi.org/10.1111/joor.12361

4. Jung F (1952) Elasticity of the bony structure of the juvenile dental system. Fortschr Kieferorthop 13:56-62. https://doi.org/10.1007/ BF02170685

5. McDowell JA, Regli CP (1961) A quantitative analysis of the decrease in width of the mandibular arch during forced movements of the mandible. J Dent Res 40:1183-1185

6. Burch JG, Borchers G (1970) Method for study of mandibular arch width change. J Dent Res 49:463. https://doi.org/10.1177/ 00220345700490025101

7. Goodkind RJ, Heringlake CB (1973) Mandibular flexure in opening and closing movements. J Prosthet Dent 30:134-138. https:// doi.org/10.1016/0022-3913(73)90046-2

8. Omar R, Wise MD (1981) Mandibular flexure associated with muscle force applied in the retruded axis position. J Oral Rehabil 8:209221. https://doi.org/10.1111/j.1365-2842.1981.tb00495.x

9. Gates GN, Nicholls JI (1981) Evaluation of mandibular arch width change. J Prosthet Dent 46:385-392. https://doi.org/10.1016/00223913(81)90443-1

10. Richter EJ (1999) Prostheses in the mandible. The in-vivo measurements of mandibular deformation and the consequences for implant-anchored suprastructures. Schweiz Monatsschr Zahnmed 109:116-134

11. Regli CP, Kelly EK (1967) The phenomenon of decreased mandibular arch width in opening movements. J Prosthet Dent 17:49-53. https://doi.org/10.1016/0022-3913(67)90050-9

12. Shinkai RS, Canabarro Sde A, Schmidt CB, Sartori EA (2004) Reliability of a digital image method for measuring medial mandibular flexure in dentate subjects. J Appl Oral Sci 12:358-362. https://doi.org/10.1590/s1678-77572004000400020

13. Keul C, Guth JF (2020) Accuracy of full-arch digital impressions: an in vitro and in vivo comparison. Clin Oral Investig 24:735-745. https://doi.org/10.1007/s00784-019-02965-2

14. Kuhr F, Schmidt A, Rehmann P, Wostmann B (2016) A new method for assessing the accuracy of full arch impressions in patients. $\mathrm{J}$ Dent 55:68-74. https://doi.org/10.1016/j.jdent.2016.10.002

15. Schmidt A, Klussmann L, Wostmann B, Schlenz MA (2020) Accuracy of digital and conventional full-arch impressions in patients: an update. J Clin Med 9. https://doi.org/10.3390/ jem9030688

16. Kamimura E, Tanaka S, Takaba M, Tachi K, Baba K (2017) In vivo evaluation of inter-operator reproducibility of digital dental and conventional impression techniques. PLoS One 12:e179188. https://doi.org/10.1371/journal.pone.0179188

17. DIN - German Institute for Standardization (2002) Rolling bearings - balls for rolling bearings and general industrial use

18. International Organization for Standardization (2014) Rolling bearings - balls - Part 1: Steel balls

19. Müller P, Ender A, Joda T, Katsoulis J (2016) Impact of digital intraoral scan strategies on the impression accuracy using the TRIOS Pod scanner. Quintessence Int 47:343-349. https://doi. org/10.3290/j.qi.a35524

20. International Organization for Standardization (1994) Accuracy (trueness and precision) of measurement methods and results Part 1: General principles and definitions

21. Kühnel S-M, Krebs D (2006) Statistics for the social sciences. Basics, methods, applications. Rowohlt, Hamburg
22. Kameyama A, Asami M, Noro A, Abo H, Hirai Y, Tsunoda M (2011) The effects of three dry-field techniques on intraoral temperature and relative humidity. J Am Dent Assoc 142:274-280. https://doi.org/10.14219/jada.archive.2011.0166

23. Ender A, Mehl A (2013) Influence of scanning strategies on the accuracy of digital intraoral scanning systems. Int J Comput Dent $16: 11-21$

24. Passos L, Meiga S, Brigagao V, Street A (2019) Impact of different scanning strategies on the accuracy of two current intraoral scanning systems in complete-arch impressions: An in vitro study. Int J Comput Dent 22:307-319

25. Aswani K, Wankhade S, Khalikar A, Deogade S (2020) Accuracy of an intraoral digital impression: a review. J Indian Prosthodont Soc 20:27-37. https://doi.org/10.4103/jips.jips_327_19

26. Ender A, Mehl A (2013) Accuracy of complete-arch dental impressions: a new method of measuring trueness and precision. J Prosthet Dent 109:121-128. https://doi.org/10.1016/S0022-3913(13)600281

27. Fischman B (1990) The rotational aspect of mandibular flexure. J Prosthet Dent 64:483-485. https://doi.org/10.1016/0022-3913(90) 90049-i

28. De Marco TJ, Paine S (1974) Mandibular dimensional change. J Prosthet Dent 31:482-485. https://doi.org/10.1016/0022-3913(74) 90169-3

29. Fischman BM (1976) The influence of fixed splints on mandibular flexure. J Prosthet Dent 35:643-647. https://doi.org/10.1016/00223913(76)90321-8

30. Prasad M, Hussain MZ, Shetty SK, Kumar TA, Khaur M, George SA, Dalwai S (2013) Median mandibular flexure at different mouth opening and its relation to different facial types: a prospective clinical study. J Nat Sci Biol Med 4:426-430. https://doi.org/10.4103/ 0976-9668.117028

31. Canabarro Sde A, Shinkai RS (2006) Medial mandibular flexure and maximum occlusal force in dentate adults. Int J Prosthodont 19: $177-182$

32. Custodio W, Gomes SG, Faot F, Garcia RC, Del Bel Cury AA (2011) Occlusal force, electromyographic activity of masticatory muscles and mandibular flexure of subjects with different facial types. J Appl Oral Sci 19:343-349. https://doi.org/10.1590/s167877572011005000008

33. Wolf L, Bergauer B, Adler W, Wichmann M, Matta RE (2019) Three-dimensional evaluation of mandibular deformation during mouth opening. Int J Comput Dent 22:21-27

34. Hobkirk JA, Schwab J (1991) Mandibular deformation in subjects with osseointegrated implants. Int J Oral Maxillofac Implants 6: 319-328

35. Abdel-Latif HH, Hobkirk JA, Kelleway JP (2000) Functional mandibular deformation in edentulous subjects treated with dental implants. Int J Prosthodont 13:513-519

36. Al-Sukhun J, Kelleway J (2007) Biomechanics of the mandible: Part II. Development of a 3-dimensional finite element model to study mandibular functional deformation in subjects treated with dental implants. Int J Oral Maxillofac Implants 22:455-466

37. Chen DC, Lai YL, Chi LY, Lee SY (2000) Contributing factors of mandibular deformation during mouth opening. J Dent 28:583588. https://doi.org/10.1016/s0300-5712(00)00041-5

Publisher's note Springer Nature remains neutral with regard to jurisdictional claims in published maps and institutional affiliations. 